\title{
Pascal et la Dimension d'un solide formé par le moyen d'une spirale autour d'un cône
}

\section{Claude Merker}

\section{OpenEdition}

\section{Journals}

Édition électronique

URL : http://journals.openedition.org/ccibp/478

DOI : $10.4000 /$ ccibp. 478

ISSN : 2493-7460

Éditeur

Centre international Blaise Pascal

Édition imprimée

Date de publication : 18 décembre 2009

Pagination : 6-16

ISBN : 978-2-84516-434-5

ISSN : 0249-6674

Référence électronique

Claude Merker, " Pascal et la Dimension d'un solide formé par le moyen d'une spirale autour d'un cône », Courrier du Centre international Blaise Pascal [En ligne], 31 | 2009, mis en ligne le 30 novembre 2015, consulté le 01 mai 2019. URL : http://journals.openedition.org/ccibp/478 ; DOI : 10.4000/ccibp.478

Ce document a été généré automatiquement le 1 mai 2019.

Centre international Blaise Pascal 


\title{
Pascal et la Dimension d'un solide formé par le moyen d'une spirale autour d'un cône
}

\author{
Claude Merker
}

1 Les quatre Lettres de A. Dettonville, pseudonyme de Pascal, contiennent l'œuvre de l'auteur en matière de calcul intégral. La Lettre I, (les sept traités sur la roulette), la plus étendue, et, à juste titre, la plus célèbre, contient des résultats très profonds, dont on a peine à réaliser qu'ils aient pu être démontrés avant l'algorithme du calcul intégral. Pourtant la méthode des indivisibles, profondément repensée, a suffi à Pascal pour surmonter toute la difficulté mathématique des problèmes de la roulette. Le tout est écrit dans une langue incomparable, qui non seulement agrée aux lecteurs, mais encore se pense elle-même en fabriquant un système littéraire d'abréviation du discours sans lequel Pascal n'aurait pu écrire cet ensemble très complexe et très articulé de traités sur la roulette.

2 Le texte de Pascal Dimension d'un solide formé par le moyen d'une spirale autour d'un cône (en abrégé : Solide spiral) dont il va s'agir ici fait partie, avec deux autres traités indépendants, de la Lettre III. Il est subordonné à la Lettre I, mais de manière presque invisible et son langage, à une exception près, pourrait même inciter à le rattacher à une conception antérieure des indivisibles.

Il s'agit de trouver le volume d'un objet géométrique, c'est-à-dire de rapporter ce volume à un autre déjà connu qui sert alors de référence, comme cela se faisait en ces temps où le calcul intégral était enraciné dans la géométrie. L'objet en question, un solide formé par le moyen d'une spirale autour d'un cône est baroque, il a plus à voir avec la diagonale qu'avec la symétrie et ne se dissocie pas, pour l'esprit qui cherche à se le représenter, des mouvements qui l'engendrent. La spirale, qui s'enroule autour d'un cône, est dans l'espace, mais elle n'est qu'un moyen, puisqu'une ligne ne saurait constituer un solide. Entre aussi en jeu un cylindre édifié non pas, comme c'est la coutume, sur un cercle, ou à la rigueur sur une ellipse, mais sur une spirale, plane celle-là. Des rectangles cylindriques servent d'instruments au calcul. Tout est inhabituel. 


\section{Le texte de Pascal}

4 Avant d'en penser quoi que ce soit, il faut voir ce qu'il dit. Comme il est très court, à peine plus de deux pages, nous allons le citer en entier, paragraphe par paragraphe, et en faire une explication détaillée.

\section{Présentation de la spirale spatiale et du solide qui s'ensuit}

Une spirale d'Archimède résulte d'un double mouvement : un point se meut sur un rayon, pendant que celui-là tourne, les deux mouvements sont uniformes et réglés l'un sur l'autre, plus précisément le point arrive à l'extrémité du rayon au moment où ce dernier achève le tour complet. Le point décrit la première volute d'une spirale.

Pascal introduit un troisième mouvement, uniforme et réglé sur les deux autres, qui fait monter le point, et donne naissance à une spirale dans l'espace cette fois. Laissons-lui la parole :

Soit un cercle donné $\mathrm{ABCD}$, dont $\mathrm{A}$ soit le centre, et $\mathrm{AB}$ un demi-diamètre; soit $\mathrm{BG}$ perpendiculaire au plan du cercle, de quelque grandeur que ce soit, par exemple égale à $\mathrm{AB}$ et soit entendu en un même temps, la ligne $\mathrm{AB}$ se tourner uniformément à l'entour du centre $\mathrm{A}$, et la ligne $\mathrm{BG}$ se porter en même temps et par un mouvement uniforme le long du demi-diamètre $\mathrm{BA}$; et soit encore entendu en même temps le point $B$ monter uniformément vers $G$; en sorte qu'en un même temps le point $\mathrm{B}$ arrive à l'extrémité de la ligne $\mathrm{BG}$, la ligne $\mathrm{BG}$ au centre $\mathrm{A}$, et le demi-diamètre $A B$ au point $B$ d'où il était parti. Par ces mouvements, la ligne $B G$ décrira une spirale BIHA dans le plan du cercle; et le point $\mathrm{B}$, en montant, décrira une espèce de spirale en l'air, ou autour d'un cône, $\mathrm{BFE}$, qui se terminera au point $\mathrm{E}$, d'où la perpendiculaire $\mathrm{AE}$ est égale à $\mathrm{BG}$.

7 Les dessins seront faits d'après celui de l'édition J. Chevalier, plus lisible que celui de l'édition originale, mais avec des suppressions ou des ajouts s'ils facilitent la compréhension.

On voit sur le dessin ${ }^{1}$ :

- La spirale dans l'espace, spirale « en l'air » comme dit Pascal, BSFE. Elle apparaît en traits gras.

- La spirale d'Archimède, BIHA, «à terre », qualifions la comme cela, eu égard au contexte.

- La surface cylindracée dont Pascal va parler incessamment, cylindre inhabituel édifié sur la spirale à terre. Trois de ses génératrices sont représentées, SI, FH correspondant à la moitié du parcours, et EA qui est la génératrice de fin de parcours.

- Le cône - habituel, lui -, qu'il faut se représenter mentalement comme édifié sur le grand cercle (centré en $\mathrm{A}$ et de rayon $\mathrm{AB}$ ), et dont $\mathrm{BE}$ est une génératrice tandis que $\mathrm{AE}=\mathrm{BA}$ est l'axe.

9 La spirale spatiale est à l'évidence dessinée sur la surface cylindrique (cylindracée) : son point courant est toujours sur une génératrice. Mais elle est aussi dessinée sur le cône : en effet un point est sur le cône quand sa hauteur est la même que la distance de sa projection à l'extrémité du rayon qui la porte. C'est bien vrai pour le point courant de la spirale en l'air puisque les mouvements de montée et de déplacement de la projection sur le rayon sont les mêmes. 
10 En résumé, cette spirale d'un nouveau genre est l'intersection du cylindre et du cône, en particulier elle est bien ce que dit Pascal « une spirale autour d'un cône ».

11 Cette nouvelle courbe n'est pas considérée pour elle-même, car sa destinée est de donner naissance à un solide, le « solide spiral » dont Pascal veut calculer la dimension, c'est-àdire, rapporter le volume à quelque volume déjà connu, une sphère, ici.

On demande, dit Pascal,

la proportion de la sphère, dont le cercle donné est un grand cercle, avec le solide spiral décrit par ces mouvements et terminé par quatre surfaces : la spirale BIHA décrite dans le plan du cercle, la portion de surface conique bornée par la droite $\mathrm{BE}$ et par l'espèce de spirale $\mathrm{BFE}$, le triangle rectiligne $\mathrm{BAE}$, et la surface cylindracée décrite par BG portée autour de la spirale BHA.

« Une spirale autour d'un cône »

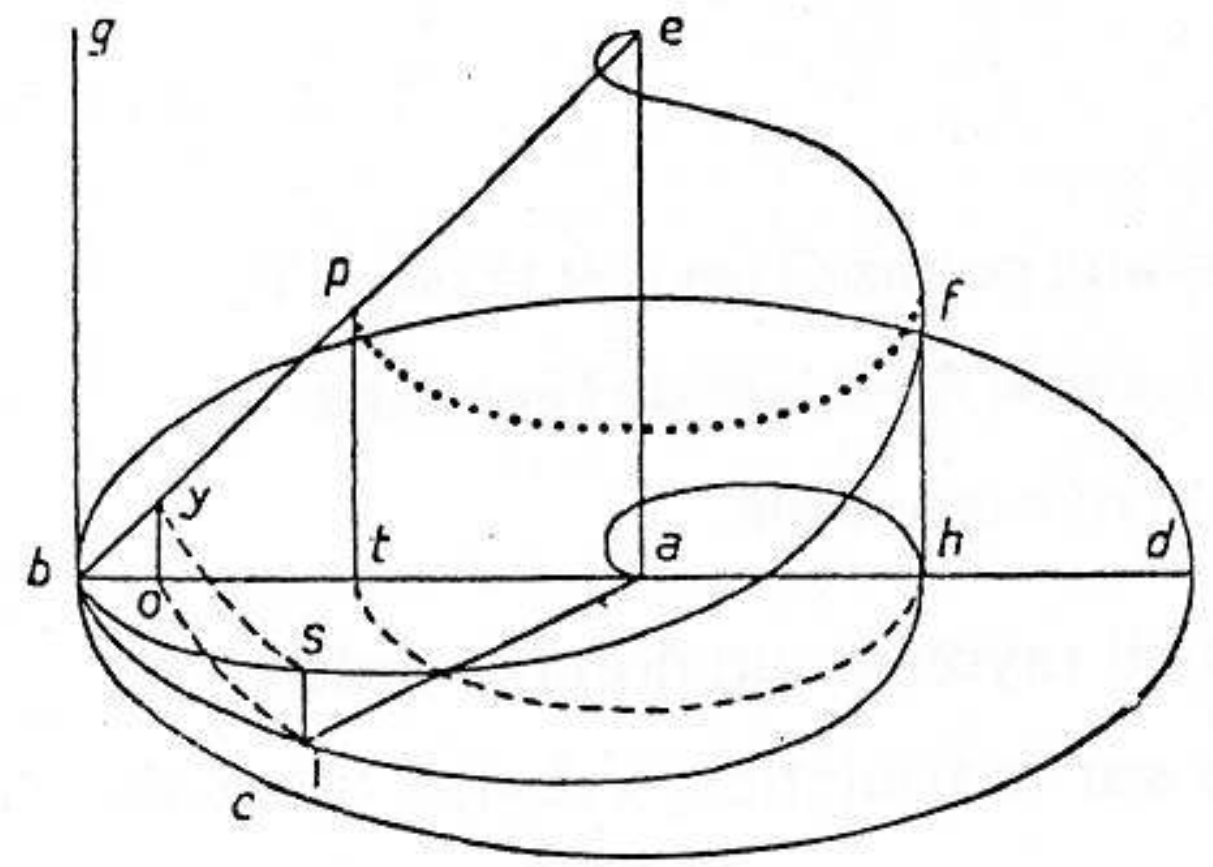

DESSIN FAIT D'APRÈS L'ÉDITION J. CHEVALIER 


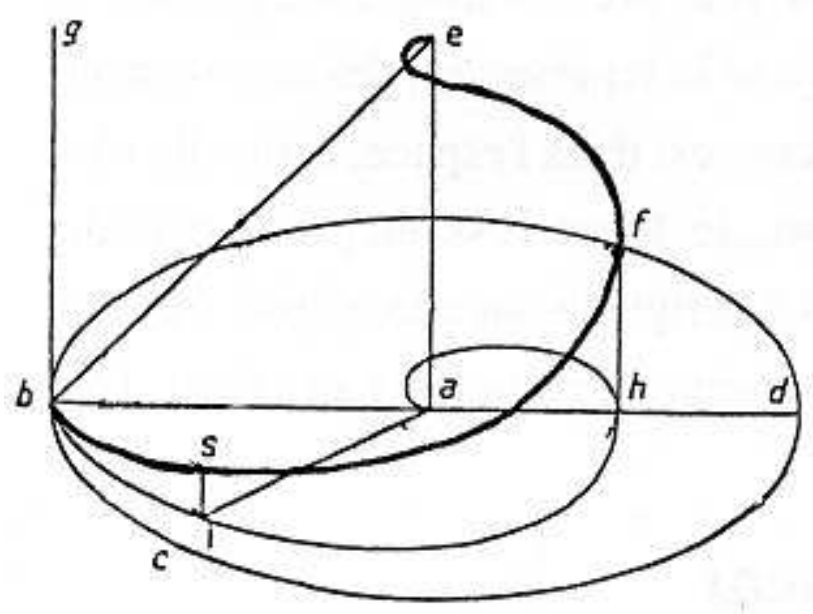

DESSIN FAIT D'APRÈS L'ÉDITION J. CHEVALIER

Représenter ce solide complètement dépourvu de symétrie est difficile, d'ailleurs Pascal ne l'a pas fait. Pour voir les quatre surfaces qui le bornent, nous avons intérêt à descendre un peu dans le texte de la solution, là où Pascal se livre à une dissection : un rectangle cylindrique, découpé dans le solide (indivisible à la manière de Cavalieri, donc) va nous faciliter la représentation, intellectuelle du moins, de la chose. Le rectangle cylindrique OYSI par son déplacement continu le long du rayon constituerait le solide, et il est clair alors que ses quatre côtés engendreraient par leur mouvement les quatre surfacesfrontières. Tout devient visible : Le segment OI engendre la spirale BIHA, OY le triangle rectiligne $^{2}$ BAE, SI la partie de la surface cylindracée qui se trouve sous la spirale spatiale, et enfin YS une portion de surface conique, sorte de di-angle, enfermée entre deux lignes dessinées sur le cône : BE et la spirale en l'air, c'est exactement ce que dit Pascal.

Ainsi en partant du bas, le solide coïncide avec la surface cylindracée (le cylindre édifié sur la spirale à terre), jusqu'à hauteur de la spirale spatiale, et à partir de là le cône prend le relais.

Pour parachever la description de ce solide surprenant, regardons évoluer son rectangle cylindrique constituant, OYSI : au départ, quand $\mathrm{O}$ est en $\mathrm{B}$, le rectangle se réduit au point $\mathrm{B}$; quand $\mathrm{O}$ devient $\mathrm{T}$, OYSI devient TPFH et c'est le dernier moment où le rectangle est devant le plan BAE; dès que $O$ dépasse $T$, le rectangle cylindrique cesse d'être entièrement devant le plan $\mathrm{BAE}$, plus précisément, le point I commence à décrire l'autre partie de la spirale à terre, à savoir l'arc HA, et le rectangle cylindrique passe en partie derrière le plan défini par $\mathrm{BAE}$; quand le point $\mathrm{O}$ arrive finalement en $\mathrm{A}$, le rectangle OYSI devient le segment $\mathrm{AE}$, d'aire tout aussi nulle que le point $\mathrm{B}$ du départ.

\section{La solution ou comment Pascal calcule la dimension du solide spiral par une méthode des indivisibles plutôt latente}

Jusque-là pour se représenter la géométrie du solide, il a été très utile d'imaginer $O$ décrivant tout le segment BA, comme cela les OYSI constituent le solide. Mais cela va cesser avec la mise en œuvre du calcul : les points de BA ne seront pas tous des points $O$. 
17 La solution est très courte, très elliptique, pleine de références internes non dites concernant le calcul de l'infini, d'une dispositio admirable, nous allons essayer à la fois de l'expliquer et d'en rétablir les sous-entendus.

Soit coupée BA en un nombre indéfini de parties égales aux points 0 : et soit le point $\mathrm{T}$ celui du milieu, d'où soit mené le demi-cercle TH qui, comme il est aisé de l'entendre, coupera le diamètre prolongé en $\mathrm{H}$ au même point où arrive la spirale.

Pascal est d'une grande discrétion sur ce découpage du rayon en un nombre indéfini de parties égales, opération qui, dans la Lettre I (les traités sur la roulette), a donné lieu à de nombreux et utiles commentaires et avertissements.

Quand le point courant a décrit radialement la moitié du chemin, il a fait aussi la moitié du chemin angulaire, autrement dit il a tourné d'un demi-tour (de par la définition même de la spirale d'Archimède), il est donc en $\mathrm{H}$.

21 Remarquer que pour un nombre indéfini de points 0 , Pascal dit qu'il y en a un au milieu. Ailleurs il aurait sans doute fait un commentaire du genre « il y en a un aussi près que l'on veut du milieu».

Soit sur ce demi-cercle élevée la surface cylindrique TPFH, qui coupe les surfaces qui bornent le solide et y donnent pour communes sections TPFH, qui sera composée de quatre lignes : savoir la ligne TP, qui se trouvera dans le plan BAE, la ligne $\mathrm{FH}$ dans la surface cylindracée égale à TP, le demi-cercle PF dans la surface supérieure, et le demi-cercle de la base TH égal au précédent PF, comme tout cela est évident; et ainsi la figure TPFH sera un rectangle cylindrique.

C'est la présentation du rectangle cylindrique fixe TPHF auquel tous les autres vont se référer. Il est une portion de surface cylindrique ordinaire, celle-là, puisqu'édifiée sur un cercle. Poursuivons :

Soit maintenant d'un des points $\mathrm{O}$ mené l'arc OI à l'entour du centre A, qui coupe la spirale en I, et soit élevé de même le rectangle cylindrique OYSI. Je dis (et ce le sera incontinent démontré) que ce rectangle cylindrique OYSI sera au premier PTHF comme BO carré en OA à BT carré en TA.

Le rectangle TPFH était un cas particulier de OYSI, celui où $O$ est en $T$, mais la démonstration que TPFH est un rectangle cylindrique vaut aussi pour le cas général.

Là commence le calcul géométrique, avec cette relation d'un indivisible générique lié au point $\mathrm{O}$ du solide, OYSI, avec un indivisible fixe, TPFH, du même solide. La relation s'écrirait avec le symbolisme d'aujourd'hui

$$
\frac{\mathrm{OYSI}}{\mathrm{TPHF}}=\frac{\mathrm{BO} 2 \times \mathrm{OA}}{\mathrm{BT} 2 \times \mathrm{TA}}
$$

Il ne faut pas s'y tromper, cette égalité est une infinité d'égalités, correspondant à l'infinité (l'« indéfinéité »...) de points $O$ nés du partage de $A B$ en un nombre indéfini de parties égales aux points $O$, précisément (c'est la première phrase de la solution).

La démonstration de cette relation est mise en attente, car elle ressortit à des résultats connus depuis Archimède sur la spirale plane, et Pascal qui a le souci de la dispositio, ne veut pas interrompre l'essentiel, à savoir ce qui est nouveau dans son discours.

Puis nous arrivons au cœur de la question, le calcul par les indivisibles va amener Pascal à reconnaître une ligne en perle (ainsi nommée par lui, mais découverte par Sluze). La densité de sous-entendus incite à couper ce paragraphe clé en deux. Voici déjà le début : 
Ce qui étant toujours véritable en quelque lieu que soit le point 0 , il s'ensuit que tous les rectangles cylindriques ensemble (c'est-à-dire le solide proposé) sera à celui du milieu PTHF pris autant de fois (c'est-à-dire au demi-cylindre qui a le cercle donné pour base et pour hauteur TP, qui est la moitié du demi diamètre) comme tous les $\mathrm{BO}$ carré en $\mathrm{OA}$ ensemble à $\mathrm{BT}$ carré en $\mathrm{TA}$, ou à $\mathrm{BT}$ cube pris autant de fois,

...

Certes, «tous les rectangles cylindriques ensemble» est une expression qui évoque la méthode des indivisibles de Cavalieri, mais s'il s'agissait vraiment de celle-là, tout point de $A B$ devrait recevoir un OYSI, autrement dit les points $O$ devraient envahir tout le diamètre $\mathrm{AB}$. Or il est explicitement dit ici que les points $\mathrm{O}$ naissent de divisions égales, et elles ont beau être en nombre indéfini, cette indéfinéité n'atteindra jamais tous les points de $\mathrm{AB}$, loin s'en faudra toujours.

Plus: Pascal ne dit jamais rien d'inutile, et la place de chacune de ses phrases est toujours signifiante ; sa solution commençait par cette phrase :

Soit coupée BA en un nombre indéfini de parties égales aux points $O$

Cela a dû nous tenir en éveil : il a l'intention de calculer l'infini avec sa méthode des indivisibles, bien à lui, qui est en réalité une méthode à différentielles géométriques : les OO, nés de divisions égales, précisément.

31 Il faut savoir que la Lettre III, dans laquelle se trouve ce traité sur le solide spiral, a été publiée à peu près en même temps que la Lettre I (les traités de la roulette ${ }^{3}$ ), magistral exposé théorique et pratique d'un calcul des indivisibles qui n'a plus guère en commun avec celui de Cavalieri que le nom. En particulier ils diffèrent en ce sens que les indivisibles de Cavalieri ont une dimension de moins que la grandeur dans laquelle ils sont découpés, tandis que ceux de Pascal lui sont homogènes, mais ce n'est pas là toute la différence, loin s'en faut ${ }^{4}$.

Il est indispensable - pour comprendre l'importance de cette phrase liminaire "Soit coupée BA en un nombre indéfini de parties égales aux points 0 »- de se rendre dans la Lettre à Carcavy, premier des sept traités de la Lettre I, où Pascal explique très clairement ce qu'il entend par somme des lignes ou somme des plans :

De sorte que quand on parle de la somme d'une multitude indéfinie de lignes ${ }^{5}$, on a toujours égard à une certaine droite, par les portions égales et indéfinies de laquelle elles soient multipliées. Mais quand on n'exprime point cette droite (par les portions égales de laquelle on entend elles soient multipliées) il faut sous-entendre que c'est celle des divisions de laquelle elles sont nées, [.... $]^{6}$

Bien que tous les rectangles cylindriques ensemble soit un vocabulaire à la Cavalieri ${ }^{7}$ la présence des divisions égales en nombre indéfini aux points $O$ oblige à voir que cette expression de Pascal désigne la somme des rectangles OYSI, au sens où Pascal emploie le mot somme, qui est toujours, il le dit, un abrégé du discours, et qui désigne ici la somme des rectangles OYSI multipliés chacun par la petite portion OO. En définitive l'objet tous les rectangles cylindriques ensemble n'est autre que la somme $\Sigma$ OYSI x OO, si nous nous autorisons la notation anachronique $\Sigma$.

4 Et chaque fois qu'il y aura tous ensemble, ou quelque chose d'analogue, il y aura des sousentendus à rétablir dans le calcul : les 00 (nés de ..., en nombre indéfini...).

Restés en place, et habillés d'une épaisseur OO, ces rectangles cylindriques OYSI constituent bien le solide, nous l'avons là, sous les yeux, géométriquement. Il en va autrement pour le «rectangle du milieu PTHF pris autant de fois» car ce solide 
irreprésenté est irreprésentable. ${ }^{8}$ La somme $\Sigma$ PTHF x 00 est un pur objet de calcul, c'est PTHF $\mathrm{x} \Sigma 00$, soit

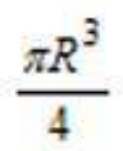

(immédiat) et c'est bien la valeur que lui donne Pascal dans la parenthèse.

Passons au calcul proprement dit. De

$$
\frac{\text { OYSI }}{\text { TPHF }}=\frac{\mathrm{BO}^{2} \times \mathrm{OA}}{\mathrm{BT}^{2} \times \mathrm{TA}}
$$

on tire (mais Pascal a sauté cette étape) en multipliant tout par 00, l'égalité tout aussi irréfutable :

$$
\frac{\text { OYSI OO }}{\text { TPHF OO }}=\frac{\mathrm{BO}^{2} \times \text { OA OO }}{\mathrm{BT}^{2} \times \text { TA OO }}
$$

Pascal a écrit directement, abruptement, par un double escamotage virtuose aussi bien de ce qui est arithmétique que de ce qui touche à l'infini :

$$
\frac{\sum \text { OYSI } \mathrm{x} \text { OO }}{\sum \text { PTHF } \mathrm{x} \text { OO }}=\frac{\sum \mathrm{BO}^{2} \mathrm{x} \text { OA OO }}{\sum \mathrm{BT}^{2} \mathrm{x} \text { TA OO }}
$$

Remarquons que cette égalité avec des $\Sigma$ ne peut découler de l'infinité d'égalités précédente que parce que TPHF et BT carré x TA sont des constantes. Autrement aucun calcul des proportions n'aboutirait à cela. La structure est :

$$
\frac{\mathrm{a}_{\mathrm{i}}}{\mathrm{c}}=\frac{\mathrm{b}_{\mathrm{i}}}{\mathrm{k}} \Rightarrow \frac{\mathrm{a}_{\mathrm{i}}}{\mathrm{b}_{\mathrm{i}}}=\frac{\mathrm{c}}{\mathrm{k}} \Rightarrow \frac{\Sigma \mathrm{a}_{\mathrm{i}}}{\Sigma \mathrm{b}_{\mathrm{i}}}=\frac{\sum \mathrm{c}}{\Sigma \mathrm{k}}
$$

de laquelle découle, par Euclide, que cette liste de fractions toutes égales entre elles est encore égale à la fraction dont le numérateur est la somme des $a_{i}$, et le dénominateur la somme des $b_{i}$ :

$$
\frac{\Sigma \mathrm{a}_{\mathrm{i}}}{\Sigma \mathrm{b}_{\mathrm{i}}}=\frac{\Sigma \mathrm{c}}{\Sigma \mathrm{k}}
$$

Finalement, en échangeant de nouveau les moyens :

$$
\frac{\mathrm{a}_{\mathrm{i}}}{\mathrm{c}}=\frac{\mathrm{b}_{\mathrm{i}}}{\mathrm{k}} \Rightarrow \frac{\Sigma \mathrm{a}_{\mathrm{i}}}{\Sigma \mathrm{c}}=\frac{\Sigma \mathrm{b}_{\mathrm{i}}}{\Sigma \mathrm{k}}
$$

L'audace, bien du XVII ${ }^{e}$ siècle, est de faire comme si la liste était finie, alors qu'elle est indéfinie (infinie...). À ce point précis on contrevient franchement à l'esprit finitiste 
euclidien. Ce qui est propre à Pascal, c'est de ne pas tenter une justification, aussi impossible à cette époque qu'inutile de son point de vue, puisque c'est évident.

Une remarque pour finir : «[...] tous les BO carré en OA ensemble » est un objet hors du monde sensible, puisqu'il est de dimension 4. Il en est de même pour «BT carré en TA, ou à BT cube pris autant de fois ${ }^{9} "$.

Et ce paragraphe clé se termine :

c'est-à-dire comme la perle du troisième ordre au rectangle de l'axe et de l'ordonnée du milieu; laquelle raison Monsieur de Sluze a donnée, non seulement dans la perle du $3^{\circ}$ ordre, mais encore dans celle de tous les ordres, où cette raison est toujours comme nombre donné à nombre donné.

Récapitulons : Pascal en est arrivé à l'égalité :

\section{$\frac{\sum \text { OYSI } \mathrm{x} \text { OO }}{\sum \text { PTHF } \mathrm{x} \mathrm{OO}}=\frac{\sum \mathrm{BO}^{2} \times \mathrm{OA} \mathrm{OO}}{\sum \mathrm{BT}^{2} \mathrm{x} \text { TA OO }}$}

- $\Sigma$ OYSI x OO est ce que l'on cherche, le volume du solide spiral.

- $\Sigma$ PTHF x OO est un volume donné, déjà calculé, dans une parenthèse plus haut.

- $\Sigma$ BT2 x TA 00 est un volume (à 4 dimensions) donné, aussi.

- $\Sigma$ BO2 x OA OO n'est pas donné, mais Pascal reconnaît là, au carré du rayon près, une figure étudiée par Sluze, la perle du troisième ordre. Plus précisément, cette perle a pour équation $\mathrm{y}=\mathrm{x}^{2}(\mathrm{~b}-\mathrm{x}) / \mathrm{b}^{2}$. Nous ne sommes pas trop anachroniques, Sluze est adepte de la géométrie analytique). Ici, b est non pas la lettre $\mathrm{B}$ du dessin de Pascal, mais le rayon, tandis que $\mathrm{x}$ est $\mathrm{BO}$, d'où $\mathrm{OA}$ est $(\mathrm{b}-\mathrm{x})$. Sluze a trouvé que l'aire de cette ligne en perle est

$$
\frac{b^{2}}{12}
$$

Le calcul est fini, (ou plutôt il restera à voir l'égalité différée) :

Donc le solide proposé est au demi-cylindre du cercle donné et de la hauteur TP en raison donnée ; donc il est aussi en raison donnée au cylindre entier de même base et de la hauteur quadruple, savoir du diamètre entier BD, et par conséquent à la sphère qui est les deux tiers du cylindre.

La raison du solide spiral au demi-cylindre vient d'être donnée, de par le résultat de Sluze, c'est $2 / 3$, celle du demi-cylindre au cylindre de hauteur quadruple est évidemment $1 / 8$, quant à celle du cylindre à la sphère, elle est $3 / 2$ par Archimède. En multipliant ces trois raisons, Pascal obtiendrait celle de son solide à la sphère : $1 / 8$ ce dont il se moque complètement, la seule chose qui lui importe est que maintenant la raison soit connue ${ }^{11}$.

Venons-en à l'égalité différée :

Or, que le rectangle cylindrique YOIS soit au rectangle cylindrique PTHF comme BO carré en $\mathrm{OA}$ à $\mathrm{BT}$ carré en TA, cela se prouve ainsi :

Je dis premièrement que l'arc OI est à l'arc TH comme le rectangle $\mathrm{BO}, \mathrm{OA}$, au rectangle $\mathrm{BT}, \mathrm{TA}$; car ces arcs $\mathrm{OI}$, TH sont en raison composée des demi-diamètres $\mathrm{AO}, \mathrm{AT}$, et des angles, ou des arcs $\mathrm{BC}, \mathrm{BCD}$, qui sont par la nature de la spirale, comme $\mathrm{CI}$ ou $\mathrm{BO}$ à $\mathrm{DH}$ ou $\mathrm{BT}$; donc ces arcs sont en raison composée de $\mathrm{BO}$ à $\mathrm{BT}$ et de $\mathrm{OA}$ à $\mathrm{TA}$, c'est-à-dire comme le rectangle $\mathrm{BO}, \mathrm{OA}$, au rectangle $\mathrm{BT}$, TA.

Dessinons pour elle-même la spirale à terre du dessin de Pascal. Le point I en est un point quelconque, et le point $\mathrm{H}$ un point fixé correspondant à un demi-tour du rayon. Le point qui engendre la spirale avance sur le rayon comme le rayon tourne, c'est la « nature de la 
spirale » (sa définition). Le point $\mathrm{B}$, initial s'est déplacé de $\mathrm{CI}$ sur le rayon, quand celui-ci a tourné d'un angle repérable par l'arc $\mathrm{BC}$, tandis que ce même point $\mathrm{B}$ s'est déplacé de $\mathrm{DH}$ sur le rayon quand ce dernier a tourné d'un angle repérable par l'arc BCD. Dans l'égalité

$$
\frac{\mathrm{IC}}{\mathrm{HD}}=\frac{\operatorname{arc~BC}}{\operatorname{arc~BCD}}
$$

est donc exprimée toute l'essence de la spirale.

Le reste coule de source. Aujourd'hui nous dirions que l'arc est le produit du rayon par l'angle au centre, mais Pascal ne mesure pas les angles, ni rien d'autre d'ailleurs; il raisonne et parle en termes de raisons (rapports), dans la tradition euclidoarchimédienne.

La raison de arc OI à arc TH est le produit des deux raisons (est en raison composée de) :

$$
\frac{\mathrm{AO}}{\mathrm{AT}} \text { et } \frac{\operatorname{arcBC}}{\operatorname{arcBCD}} \text {. }
$$

D’où

$$
\frac{\operatorname{arc~OI}}{\operatorname{arc~} \mathrm{TH}}=\frac{\mathrm{AO}}{\mathrm{AT}} \times \frac{\operatorname{arcBC}}{\operatorname{arcBCD}}=\left(\text { nature de la spirale) } \frac{\mathrm{AO}}{\mathrm{AT}} \times \frac{\mathrm{IC}}{\mathrm{HD}}=\frac{\mathrm{AO}}{\mathrm{AT}} \times \frac{\mathrm{BO}}{\mathrm{BT}}\right.
$$

Passer des arcs aux rectangles cylindriques coule également de source.

Venons-en maintenant aux rectangles cylindriques YOIS, PTHF. Il est visible qu'ils sont en raison composée des hauteurs et des bases, c'est-à-dire en raison composée de OY à TP, ou BO à BT [...]

Il est inutile de continuer, il n'y a qu'à multiplier l'égalité de rapports précédentes par encore une autre (que de raisons composées !), pour avoir le résultat final.

Pascal ne s'étend pas sur la surface d'un rectangle cylindrique, il est bien évident pour lui qu'un tel rectangle, bien que cylindrique, est parfaitement plat, et il n'a même pas besoin de mentionner quoi que ce soit à ce sujet.

Les solides des autres spirales des ordres supérieurs se trouveront de même par le moyen des lignes en perle des ordres supérieurs.

Telle est la fin de ce texte court et dense. Nous faisons confiance à Pascal, pour la réussite de ce programme qui consistera à faire tourner des lignes en perle de degré supérieur pour générer des spirales d'ordres supérieurs.

\section{Affinités de la spirale d'Archimède et de la parabole, ce qui en découle ici}

L'idée du solide spiral, dont on ne peut pas dire qu'elle s'impose naturellement à l'esprit, est venue à Pascal à la fois des lignes en perle de Sluze et de l'affinité que la spirale entretient avec la parabole ${ }^{12}$. Une perle d'ordre $n+1$ est une courbe d'équation

$$
y=\frac{x^{n}(b-x)}{b^{n}}
$$

Voir qu'il s'agissait de courbes déjà connues (paraboles généralisées) n'a pas été facile à l'époque, car pour cela il faut faire quelque chose qui s'apparente à un changement de 
variable. Revenons à la perle d'ordre 3, celle que nous avons rencontrée dans le calcul de Pascal, ci-dessus.

Sluze écrit à Pascal (c'était le 29 Juin 1658) :

[...] et je vous suis bien obligé que vous m'ayez fait part de votre intention très subtile touchant les cylindres tournés en spirale. J'avais bien remarqué que des perles on en pouvait faire des spirales en tournant leurs appliquées en arc de cercle [...] Mais je n'avais jamais eu la pensée d'y joindre un troisième mouvement perpendiculaire pour composer le solide dont vous avez si doctement démontré la mesure.

En 1658 les affinités entre spirale et parabole étaient déjà repérées depuis longtemps. En effet, en 1625, Grégoire de Saint-Vincent, mû par son désir de trouver la quadrature du cercle, et très impressionné par le résultat d'Archimède concernant la tangente à la spirale, qui, espérait-il, l'amènerait à la transmutation tant désirée, s'était lancé dans une étude de cette courbe et avait montré que «la spirale d'Archimède est une parabole enroulée sur elle-même, et que réciproquement la parabole est une spirale déroulé ${ }^{13}$ ». Le contentement qu'il en a prouve que cette accointance n'était pas connue. De même Cavalieri avait, dans la Geometria indivisibilibus, ouvrage publié en 1635, déroulé les arcs de cercle finissant sur la spirale et vu qu'alors les extrémités dessinaient une parabole.

Dans la langue mathématique d'aujourd'hui qui dispose de la mesure des angles, et ne se soucie plus du tout d'homogénéité, la chose est devenue presque évidente, en effet, l'arc de cercle terminé à la spirale étant - comme tout arc - le produit de son rayon par son angle, si l'angle est égal au rayon, alors l'arc devient le carré du rayon, il n'y a plus qu'à le dérouler pour voir apparaître immédiatement la parabole classique, celle de degré 2 . En somme en enroulant les ordonnées d'une parabole, les extrémités dessinent une spirale, et inversement en déroulant les arcs de cercle extérieurs à une spirale, les extrémités dessinent une parabole.

Spirale et parabole
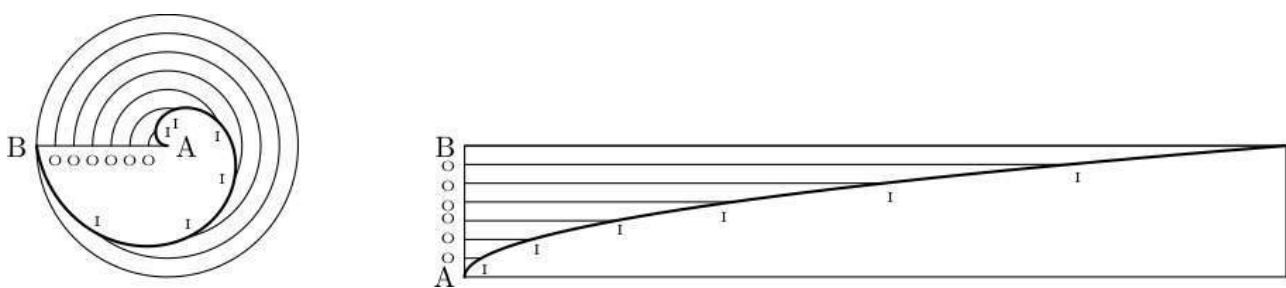

Ainsi, dans le cas - le plus simple - où le point part de l'origine, l'équation $\mathrm{q}=\mathrm{r}$ décrit la nature de la spirale (le point avance sur le rayon comme celui-là tourne); l'égalité Arc $\mathrm{OI}=r q$, devient $\mathrm{Arc} \mathrm{OI}=\mathrm{r}^{2}$, qui exprime que I est sur la spirale. Une fois les arcs OI déroulés, les points I dessinent une parabole d'« équation » $\mathrm{OI}=\mathrm{AO}^{\mathrm{z}}\left(\mathrm{y}=\mathrm{x}^{2}\right)$.

Dans le texte de Pascal la seule différence est que le point part de l'extrémité du rayon. Autrement dit l'angle n'est pas le rayon du point variable, mais son complément au rayon fixe du grand cercle. C'est donc BO x AO, qui est la longueur de l'arc variable OI, intérieur à la spirale à « terre » (AO est le rayon de l'arc et BO représente l'angle). 


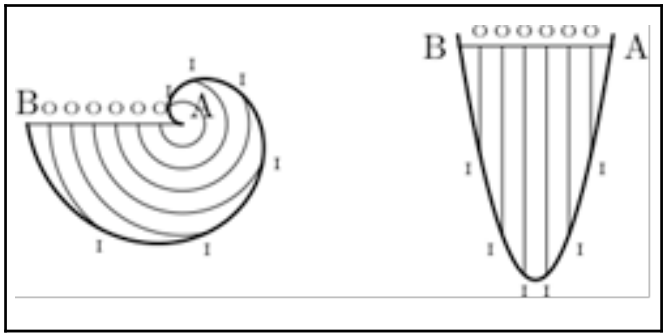

61 Si $b$ désigne maintenant le rayon $A B$, la spirale à terre que considère Pascal a pour équation moderne $r=b-q$, puisque le point part de $B$, et non pas du centre. Du coup les arcs de cercle OI qui, chez Cavalieri remplissaient l'extérieur de la spirale, lui sont cette fois intérieurs, et $\mathrm{Arc} \mathrm{OI}=\mathrm{r}(\mathrm{b}-\mathrm{r})$. Une fois dépliés comme les autres, leurs extrémités I dessineront aussi une parabole $(\mathrm{y}=\mathrm{x}(\mathrm{b}-\mathrm{x}))$.

La perle de Sluze commence à se montrer, il manque un $\mathrm{r}$ (un $\mathrm{x}$, $\mathrm{x}$ n'est jamais qu'un $\mathrm{r}$ déplié) qui est justement dû au troisième mouvement. L'ascension correspondante du point courant de la spirale en l'air est $r$, et ainsi apparaît l'expression $r(b-r) r$, où les deux premiers facteurs parlent de la spirale d'Archimède et le troisième est propre à ce troisième mouvement qui est une création pascalienne. On imagine les OI dépliés, si on les multiplie par $\mathrm{r}$ (l'ascension), on obtient les rectangles cylindriques redressés, et c'est là que Pascal « reconnaît » la perle du troisième ordre. Il ne faut pas s'y tromper, c'est $\mathrm{x}$ $(b-x)$ qui parle des accointances de la spirale avec la parabole, l'autre $x$ ne parle que du mouvement ascensionnel.

Représenter la perle du troisième ordre, par

$$
y=\frac{x(b-x) x}{b^{2}}
$$

c'est, d'entrée de jeu, avoir l'ordonnée perpendiculaire à l'abscisse, c'est donc

- redresser la base du rectangle cylindrique $\mathrm{x}(\mathrm{b}-\mathrm{x})$,

- considérer sa hauteur : $\mathrm{x}$,

- satisfaire à l'homogénéité : diviser par b².

\section{Dérouler une spirale, ou enrouler une parabole?}

Pour créer son solide spiral, Pascal a fait l'inverse, il a, comme dit Sluze « fait tourner les lignes en perle ${ }^{14}$ », et fabriqué ainsi ces rectangles cylindriques qui sont au solide spiral ce que les arcs de cercle sont à la spirale à terre.

Ainsi, la rédaction est en sens inverse de l'invention, ce n'est pas le solide qui produit par déroulement de ses rectangles cylindriques une courbe connue, sur laquelle on tomberait par un heureux hasard, mais l'enroulement de la perle qui fabrique le solide spiral, si l'on en croit Sluze.

\section{Le calcul de l'infini, relations des Lettres III et II avec la Lettre I}

Il est surprenant que Pascal soit aussi silencieux ici sur ces 00 , au point de ne même pas mentionner qu'il les sous-entend dans le calcul (il aurait suffi pour cela d'employer le mot « somme »). Alors que les 00 , ces « $\mathrm{dx}$ géométriques », sont la spécificité de sa méthode 
des indivisibles et qu'ils ont donné - avec d'autres petites portions, qui sont des «ds géométriques ${ }^{15}$ »- des résultats éblouissants dans la Lettre I, une seule ligne les mentionne ici, sans les rattacher à rien, et il n'en est plus jamais parlé. Silence complet sur la Lettre I, origine des concepts.

Qu'en est-il dans les deux autres traités de la Lettre III ? Dans celui qui concerne l'escalier, Pascal fait explicitement référence à deux propositions de la Lettre I (du le Traité des arcs), et surtout il emploie deux fois l'expression «la somme des solides [...]», avec le sens pascalien du mot « somme » (il faudra multiplier par les portions égales et indéfinies etc.). Dans celui qui concerne les triangles cylindriques, Pascal cite le traité des solides circulaires (le troisième de la Lettre I), et une proposition du Traité des sinus du quart de cercle (le quatrième de la même).

Plus, dans la Lettre II, il y a onze fois une expression comme « la somme des HL », et cinq fois sur ces onze, Pascal met entre parenthèses le rétablissement du sous-entendu, comme " multipliés par les petits arcs LL », comme pour bien nous rappeler le sens qu'il a donné au mot « somme »... Il est vrai que la Lettre II qui traite des roulettes généralisées est le prolongement naturel de la précédente, et qu'elle a été conçue tout de suite après. impliquent automatiquement une multiplication sous-entendue par les petites portions et qu'il n'est plus besoin de le rappeler.

\section{Conclusion} l'enjeu de la Lettre I est de faire entrer les lignes trigonométriques dans le sérail du calcul intégral, ce qui est nouveau et fécond, tandis que le solide spiral ne semble pas aller audelà de lui-même. De plus, du côté technique, il n'y a pas ici ce vertige des transformations d'éléments différentiels qui passent virtuosement d'un axe à l'autre ou à la courbe, et d'une courbe à une autre, à l'intérieur d'un même calcul ${ }^{17}$. Ici les 00 ne bougent pas de l'axe, et les indivisibles pascaliens à nuls autres comparables, au faîte de leur splendeur dans les traités sur la roulette, sont ici sous-employés.

Cependant, cet objet transcendant dont Pascal a calculé le volume est une pierre de plus à l'édification du calcul infinitésimal. Pascal a une prédilection pour les courbes mécaniques, la roulette, la spirale, ces courbes qui ont de l'infini dans leur définition parce qu'elle met en rapport de l'angle et de la longueur. La spirale a aussi une propriété qui suggère la structure ternaire "opinion du peuple, du demi-habile, de l'habile », car après un tour elle revient au départ (même angle), mais avec une avancée (le long du rayon).

74 À force d'explications ne finit-on pas par noyer la compréhension? Surtout pour un texte à la concision aussi savamment maitrisée. Espérons que cela reste un plaisir de lire

Courrier du Centre international Blaise Pascal, 31 | 2015 
quelqu'un qui écrit avec cet art de l'essentiel. La disposition est très pensée : présentation de l'objet géométrique, une ligne placée au début de la preuve pour en donner la première clé (soit $\mathrm{AB}$ divisé etc.), rejet de la démonstration d'une relation (entre OYSI et PTHM) à la fin dans le but de mettre en valeur l'importance du résultat de Sluze sur les lignes en perle, lequel arrive après un instantané tour de passe-passe euclido-infinitiste mélangeant étonnamment théorie des proportions et infini, absence d'achèvement du calcul qui n'apporterait rien de plus. Après avoir tout compris, on voit que c'était parfait et au fond très clair... (un peu à la manière d'un poème de Mallarmé). Pascal n'a pas renoncé ici à l'Art d'agréer.

\section{BIBLIOGRAPHIE}

H. BOSMANS S.J., « Sur l'interprétation géométrique donnée par Pascal à l'espace à quatre dimensions ", dans Annales de la Société scientifique de Bruxelles, t. XLI, 1923, $1^{\text {re }}$ partie, pp. 337-345. H. BOSMANS S.J., "Sur une contradiction reprochée à la théorie des indivisibles chez Cavalieri », ibidem, pp. 82-89.

H. BoSmanS S.J., « La notion des « indivisibles » chez Blaise Pascal », dans Archivio di Storia delle Scienze, t. IV, 1923, pp. 369-379.

D. DESCOTES Blaise Pascal. Littérature et Géométrie Presses universitaires Blaise Pascal. Spécialement pp. 317-322.

V. JULLIEN, «Les indivisibles de Roberval, une « petite différence » de doctrine, une moisson de résultats », in Géométrie, atomisme et vide dans l'école de Galilée ENS Éditions.

C. MERKER, Le chant du cygne des indivisibles, Presses universitaires Franc-Comtoises. CiD.

Spécialement pp. 89-99.

J. MESNARD, Pascal. Cuuvres complètes, Desclée de Brouwer, 4 Tomes. En abrégé OC.

C. NAUX, «L'Opus geometricum de Grégoire de Saint-Vincent », dans Revue d'histoire de sciences, 1962. Spécialement pp. 94-96.

\section{ANNEXES}

\section{Quelques réflexions sur le calcul de l'infini et la méthode des indivisibles, chez Pascal et chez Cavalieri}

Nous gardons cet objet particulier qu'est le solide spiral, avec ses surfaces OYSI découpées à son intérieur, car il peut servir de support à un discours plus général sur les difficultés qu'il y a à calculer l'infini au XVII ${ }^{\mathrm{e}}$ siècle. 
Utiliser les aires des OYSI - sans leur accorder d'épaisseur - pour calculer le volume du solide spiral poserait sérieusement question, puisque le volume de chaque OYSI est rigoureusement nul. Comment une somme de zéros pourrait-elle ne pas faire zéro ? Cette question, formulée de manière apparemment particulière, est générale et jalonne toute la période de création du calcul infinitésimal, jusqu'au XIX ${ }^{\mathrm{e}}$ siècle. Elle est déjà posée par les paradoxes de Zénon d'Elée.

Les OYSI composent le solide, mais pas en nombre dénombrable. Cette composition ressortit à la puissance du continu, et sur la valeur de $\infty$ x 0 , quand l'infini est celui du continu, les mathématiques sont muettes. L'axiome moderne de la mesure le dit très bien (sans jeu de mot), en ne parlant que de $\Sigma$-additivité dénombrable, il permet de ne pas dire que la longueur du segment [ 01 1] est nulle, au prétexte que chacun de ses points a une longueur nulle. Seule une somme dénombrable de zéros sera zéro.

Cavalieri ne prend pas parti sur la composition du continu. Il compare deux grandeurs (de même dimension) en comparant les indivisibles, d'une dimension de moins, qui y sont découpés. Si les indivisibles restent dans un rapport constant, d'une des deux grandeurs à l'autre, ce rapport se retrouve dans les grandeurs. Il faut deux grandeurs, il n'y a donc pas de calcul autonome a priori. De plus, des paradoxes surgissent si la correspondance indivisible-à-indivisible ne respecte pas des règles très strictes. Chez Cavalieri, il s'agit de comparer, plutôt que de calculer, dès lors, le mot composer peut garder un sens assez flou. S'il s'agissait de calculer, au contraire, dire que le solide est composé des OYSI déboucherait sur l'affirmation que le solide est la somme des OYSI et sur le paradoxe des zéros lorsqu'il y a puissance du continu. En revanche, un axiome bien énoncé, avec une règle parallèle découpant simultanément des indivisibles d'une dimension de moins dans deux grandeurs va toujours fournir des résultats justes.

Quant à Pascal, dans la Lettre I, il est dans le sillage de Roberval, et attribue systématiquement aux indivisibles une épaisseur. Cela revient ici à considérer la somme des OYSI x OO. Une autre difficulté surgit, bien sûr : aussi grand que soit le nombre des points $\mathrm{O}$, la somme - finie - des OYSI x OO ne sera qu'une valeur approchée du volume du solide. Dans un célébrissime avertissement de la Lettre à Carcavy (début de la Lettre I) Pascal explique très clairement pourquoi la difficulté n'en est pas une. Nous nous excusons auprès de ceux qui le connaissent par cœur de le citer une fois de plus :

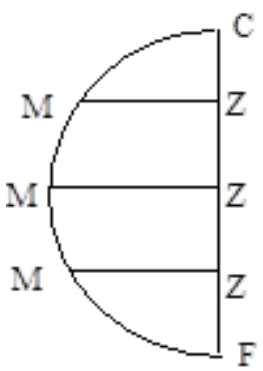

J'ai voulu faire cet avertissement pour montrer que tout ce qui est démontré par les véritables règles des indivisibles se démontrera aussi à la rigueur et à la manière des anciens; et qu'ainsi l'une de ces méthodes ne diffère de l'autre qu'en la manière de parler : ce qui ne peut blesser les personnes raisonnables quand on les a une fois averties de ce qu'on entend par là. Et c'est pourquoi je ne ferai aucune difficulté dans la suite d'user de ce langage des indivisibles, la somme des lignes ou la somme des plans; et ainsi quand je considérerai par exemple le diamètre d'un demi-cercle 
divisé en un nombre indéfini de parties égales aux points $Z$, d'où soient menées les ordonnées ZM, je ne ferai aucune difficulté d'user de cette expression, la somme des ordonnées, qui semble n'être pas géométrique à ceux qui n'entendent pas la doctrine des indivisibles, et qui s'imaginent que c'est pécher contre la géométrie que d'exprimer un plan par un nombre indéfini de lignes; ce qui ne vient que de leur manque d'intelligence, puisqu'on n'entend autre chose par là sinon la somme d'un nombre indéfini de rectangles fait de chaque ordonnée avec chacune des petites portions égales du diamètre, dont la somme est certainement un plan, qui ne diffère de l'espace du demi-cercle que d'une quantité moindre qu'aucune donnée.(...)

En adoptant un point de vue très anachronique :

L'infini de Cavalieri ressortit à l'infini de R, c'est-à-dire à la puissance du continu : les points $\mathrm{O}$, pour lui, seraient tous les points du diamètre. Cela convient pour une méthode des indivisibles qui ne fait pas de calcul autonome, mais seulement des comparaisons.

L'infini de Pascal ressortit à celui de $\mathrm{N}$, c'est à dire à la puissance du dénombrable puisque les points $\mathrm{O}$ sont des points rationnels, les autres ne pouvant jamais être atteints par cette opération qui consiste à couper «BA en un nombre indéfini de parties égales aux points $\mathrm{O}$ ». « Indéfini » a tantôt, comme ici, un sens plutôt potentiel, (on imagine BA coupée en un nombre $\mathrm{n}$ de parties égales et $\mathrm{n}$ augmentant sans cesse), et tantôt un sens actuel, comme dans l'avertissement du Traité des sinus du quart de cercle (une certaine égalité n'est pas véritable quand la multitude est finie, néanmoins elle est véritable quand la multitude est indéfinie, dit $\mathrm{Pascal}^{18}$ ). On pourrait appeler « indéfinéité » ce concept pascalien d'infini-de-divisions qui enferme deux idées, celle de devenir et celle d'achèvement.

\section{NOTES}

1. Pascal désigne les points par des lettres minuscules sur le dessin, et par des majuscules dans le texte.

2. En général on ne précise pas qu'un triangle est rectiligne. Mais ici le contexte est différent : la deuxième partie de la Lettre III traite des triangles cylindriques, et d'autre part Pascal introduit dans cette troisième partie des rectangles cylindriques.

3. Voir J. Mesnard, $O C$, tome IV, page 425

4. Ceux de Roberval, de Torricelli, sont homogènes aussi, mais leurs "éléments différentiels" ne sont pas isolés puis théorisés, comme ils le sont par Pascal qui crée un vrai concept de différentielles (enracinées dans la géométrie). N’oublions pas d'autre part que Pascal est un théoricien du langage (voir ce qu'il dit de la liberté des définitions, de l'abrégé du discours, in Mesnard, OC, tome II, pp. 393-394).

5. La même chose vaut pour une somme indéfinie de surfaces, les OYSI, par exemple.

6. Mesnard, OC, tome IV, p. 425.

7. Qui disait omnes lineae pour les problèmes de la dimension deux (et parfois aussi, la somme des lignes, ce qui ajoute à la confusion).

8. A priori, PTHF, fixe, ne décrit rien du tout. Il faudrait donc imaginer de suspendre des PTHF tous égaux en chaque point $\mathrm{O}$ de la litanie des subdivisions (translater PTHF de manière à amener $\mathrm{T}$ en $\mathrm{O}$ ), leur accorder à chacun l'épaisseur 00 ; mais aucun solide géométrique égal à la « somme des PTHF » ne se dessine, car certains endroits de ce présumé solide appartiendraient à deux PTHF différents, comme on s'en rend compte facilement en translatant un cylindre de papier le long du rayon, de B vers A. 
9. On pourra voir ce que dit à ce propos H. Bosmans, dans son article «Sur l'interprétation géométrique donnée par Pascal à l'espace à quatre dimensions » (cf bibliographie).

10. Ce qu'un calcul intégral immédiat montre aujourd'hui.

11. Sur la manière dont Pascal identifie "connu" et "donné", on peut de reporter à la fin de la Lettre à Carcavy (premier traité de la Lettre I).

12. Il sera seulement question ici d'affinité d'aire, car il y a aussi une affinité de longueur, entre ces deux courbes, étudiée par Torricelli, Roberval, et Pascal lui-même dans la Lettre IV.

13. C'est ce que dit Charles Naux, dans son article «L'opus geometricum de Saint-Vincent ", paru dans la Revue d'Histoire des Sciences, 1962, p. 97.

14. Mesnard, $O C$, Tome IV, lettre de Sluze à Pascal pp. 124-125. Voir également la notice page 115.

15. Lorsque les petites portions $O 0$ naissent d'une courbe, et non pas d'un axe.

16. «Or dans la Lettre de M. de Carcavy à M. Dettonville sont bien mentionnées les recherches de ce dernier sur l'escalier et sur les triangles cylindriques, sujet des deux premiers chapitres, mais non le problème du solide formé par le moyen d'une spirale autour d'un cône, sujet du dernier chapitre. C'était donc là une recherche plus récente » J. Mesnard OC, Tome IV, p. 378.

17. Voir C. Merker Le chant du cygne des indivisibles, chap. III et VI.

18. Je souligne. Voici le passage en entier : «Quand j'ai dit que [...] chaque touchante EE est égale à chacun des petits arcs DD, on n'a pas dû en être surpris, puisqu'on sait assez qu'encore que cette égalité ne soit pas véritable quand la multitude des sinus est finie, néanmoins l'égalité est véritable quand la multitude est indéfinie; parce qu'alors la somme de toutes les touchantes égales entre elles, EE, ne diffère de l'arc entier BP, ou de la somme de tous les arcs égaux DD, que d'une quantité moindre qu'aucune donnée. »

INDEX

Mots-clés : Pascal, solide, spirale, mathématiques

Keywords : solid, spiral, mathematics

\section{AUTEUR}

\section{CLAUDE MERKER}

Département de mathématiques, Université de Franche-Comté, professeure agrégée 